\title{
Signal identification: The comparison of two competitive theories
}

\author{
KARL F. WENDER \\ Technische Universität Braunschweig, Institut für Psychologie \\ 93 Braunschweig, Spielmannstrasse 19, West Germany
}

\begin{abstract}
On each of a series of random trials, 10 observers had to identify which of two possible tones of different amplitude had been presented. Two theories for this paradigm of signal identification are compared. One theory, the memory-trace model, is a normal process model, whereas the competitive theory, the memory-state model, assumes high thresholds operating in a Markov chain. Different predictions for sequences of stimulus response pairs are derived. The results of the experiment involving payoffs contingent upon the previous answer strongly favor the memory-trace model.
\end{abstract}

In the simplest experimental paradigm for signal identification, the subjects is presented, on every trial, with one of two possible stimuli, $S_{1}$ and $S_{2}$. The subject tries to identify which stimulus has been presented, and responds with $A_{1}$ or $A_{2}$, respectively. Ordinarily both signals are easily detected. The difference between them is so small, however, that they are not discriminated with certainty.

Two theories have been offered to explain what is going on in this simple experiment of signal identification or signal recognition. These theories, however, come from rather different theoretical backgrounds.

Tanner, Haller, and Atkinson (1967) developed a model in the tradition of signal detection theory, whereas Sandusky (1971) advocates a threshold model combined with a homogeneous Markov process. Both theories have been found to describe data from different experiments quite satisfactorily. To quote Sandusky: "It is remarkable that two theories with such divergent bases can so closely parallel each other in terms of predicted response probabilities" (Sandusky, 1971, p. 340).

The present study again tries to decide between the two theories. As will be argued, the additional results presented here strongly favor the Tanner et al. theory.

The problem under investigation is related to a discussion in the field of human signal detection, namely, whether or not there is a sensory threshold operating in the process of detection. With respect to signal detection at least. a preliminary decision has, apparently, been reached. As has been summarized recently by Laming, threshold models such as Blackwell's (1963) or Krantz's (1969) or Luce's (1963) did not survive the accumulating contradicting evidence (Laming, 1973, p. $105 \mathrm{ff}$ ).

Similarly, in studies of recognition memory where

The research reported herein was supported by Grant WE 498/3 from the Deutsche Forschungsgemeinschaft. The assistance of Karl Hegner in running the experiment is gratefully acknowledged. lists of items to be remembered contain more than just two alternatives and where during the test phase old and new items are mingled together, strength theory has been found to be superior to threshold theories. This point was summarized recently by Murdock (1974. p. $17 \mathrm{ff})$.

The present study is concerned with the same problem with respect to the simplest form of signal recognition as described at the outset. This experimental situation is very similar to that in signal detection. The results, however, differ in at least two important points from signal detection. (1) If the probability for presenting $S_{1}$ is different in different sessions, the probability of hits and of false positives both tend to vary in the opposite direction. That is, with increasing $P\left(S_{1}\right)$, both $P\left(A_{1} \mid S_{1}\right)$ and $P\left(S_{1} \mid A_{2}\right)$ decrease, and vice versa. This has been dubbed the probability contrast. It was found by Parducci and Sandusky (1965), Sandusky (1971), and Tanner, Rauk, and Atkinson (1970). The probability contrast bears some resemblance to the word frequeney effect observed by Gorman (1961), Shepard (1967), and others. However, the exact relationship between these two effects is far from clear. (2) In signal identification, the responses apparently depend on the stimulus-response pair of the preceding trial. Such sequential dependencies have not been found in signal detection. The sequential dependencies will be analyzed in more detail below.

The following two paragraphs summarize the main features of both theories. For more detail, the reader is referred to Sandusky (1971) and Tanner et al. (1967).

\section{MEMORY TRACE MODEL}

This model assumes the following:

(T1) The effect of presenting $S_{i}$ is a normally distributed random variable, $I_{i}$, with expected value $S_{i}$, $=1,2 . I_{1}$ and $I_{2}$ have equal variances $\sigma_{I}^{2}$. The unit of 
measurement is so chosen that $S_{1}=0$ and $S_{2}=1$.

(T2) On every trial, the subject has access to a trace of the preceding signal. The trace of $S_{k}$ is an asymptotically normally distributed random variable, $T_{k} . T_{1}$ and $T_{2}$ have equal variances $\sigma_{\mathrm{T}}^{2}$. The trace on any given trial, $\mathrm{n}$, is the weighted sum of the trace and the signal effect from the preceding trial; that is,

$t_{i, n}=(1-\alpha) t_{k, n-1}+\alpha s_{i, n-1}, i=1,2$ and $0 \leqslant \alpha \leqslant 1$

The parameter $\alpha$ is the weight of the present signal compared to the old trace.

For random sequences of $S_{1}$ and $S_{2}$, it may be shown that the expected value of $T_{k}$ on trial $n$ is

$t_{k, n}= \begin{cases}(1-\alpha) \gamma \quad \text { if } S_{1} \text { was presented on } n-1 \\ (1-\alpha) \gamma+\alpha & \text { if } S_{2} \text { was presented on } n-1\end{cases}$

where $\gamma$ is the a priori probability of $S_{1}$.

(T3) On every trial, the subject judges the difference between signal and trace. Assuming independence of $S_{i}$ and $T_{k}$, the distribution of differences, $D_{i k}=S_{i}-T_{k}$, is asymptotically normal with expected value $s_{i}-t_{k}$ and variance $\sigma_{\mathrm{D}}^{2}=\sigma_{\mathrm{I}}^{2}+\sigma_{\mathrm{T}}^{2}$.

The subjects respond according to the following rule

$$
\text { if } \begin{cases}\mathrm{d}_{\mathrm{ik}}>\delta_{2} & \text { respond with } \mathrm{A}_{1} \\ \mathrm{~d}_{\mathrm{ik}}<\delta_{1} & \text { respond with } \mathrm{A}_{2} \\ \delta_{1}<\mathrm{d}_{\mathrm{ik}}<\delta_{2} & \text { repeat the last answer. }\end{cases}
$$

This model contains four free parameters. $\sigma_{D}^{2}$ may be interpreted as a sensitivity parameter, $\alpha$ determines the effect of the immediately preceding signal upon the following trace. The larger the value of $\alpha$, the more the trace on $\mathrm{n}$ is determined by the signal on $\mathrm{n}-1$. For $\alpha=0$, the trace approaches $\gamma$ independently from the immediately preceding signal. $\delta_{1}$ and $\delta_{2}$ may be looked at as response bias parameters. They have been found to depend on the a priori probabilities. The decision rule is motivated by the following consideration. If the subject experiences a large positive difference between signal and trace, the conclusion is that the stronger signal must be present. If the experienced difference has a large negative value, then the present signal must be the weak one. If the difference is not far from zero, then the last and the present signal must probably be identical, and therefore the last response is repeated.

The sequential probabilities are obtained in this model as given by Equation 5 below. Notice that $p\left(A_{1} \mid S_{i}, A_{j}\right.$, $S_{k}$ ) depends on $\delta_{j}$. Furthermore, the preceding response, $A_{j}$, determines which one of $\delta_{1}$ and $\delta_{2}$ enters into the computation. Hence, this model predicts that the response on trial $\mathrm{n}$ depends on the answer on trial $\mathrm{n}-1$ if $\delta_{1} \neq \delta_{2}$. This kind of dependency has been observed in experiments.

\section{MEMORY STATE MODEL}

This model makes the following assumptions:

(S1) Following each signal presentation, the subject is in one of three sensory states: (a) $S_{1}$ was recognized correctly, (b) $S_{2}$ was recognized correctly, or (c) no signal was recognized. This assumption qualifies the model as a double high-threshold model.

(S2) The probabilities of recognizing $S_{1}$ and $S_{2}$ are $\alpha_{1}$ and $\alpha_{2}$, respectively. If a signal is recognized, the correct answer follows with probability 1. Furthermore, $\alpha_{1}=\alpha_{2}$ $=\alpha$ is assumed.

(S3) If no signal is recognized, then the preceding response is repeated with some probability. This probability depends on the preceding sensory state. If the preceding state was a recognition state, then the probability to repeat the last response is $\rho$. If the subject was in the nonrecognition state, then the probability for repetition is $\nu$. Additionally, it is assumed that $\nu \geqslant \rho$.

The sequence of sensory states and responses is conceived as a homogeneous Markov process with four states: In $Z_{1}$, signal $S_{1}$ is recognized and the response is $A_{1}$; in $Z_{2}, S_{2}$ is recognized and $A_{2}$ is the response; in $Z_{3}$, no signal is recognized and the response is $A_{1}$; and, finally, in $Z_{4}$, no signal is recognized and $A_{2}$ is the response. The transition matrix for this process is:

$$
\mathrm{Z}_{\mathrm{Z}_{2}}\left\{\begin{array}{cccc}
\mathrm{Z}_{1} & \mathrm{Z}_{2} & \mathrm{Z}_{3} & \mathrm{Z}_{4} \\
\mathrm{Z}_{\mathbf{4}} & (1-\gamma) \alpha & (1-\alpha) \rho & (1-\alpha)(1-\rho) \\
\gamma \alpha & (1-\gamma) \alpha & (1-\alpha)(1-\rho) & (1-\alpha) \rho \\
\gamma \alpha & (1-\gamma) \alpha & (1-\alpha) \nu & (1-\alpha)(1-\nu) \\
\gamma \alpha & (1-\gamma) \alpha & (1-\alpha)(1-\nu) & (1-\alpha) \nu
\end{array}\right\}
$$

The model has three free parameters. $\alpha$ can be attributed to the sensory process. The values of $v$ and $\rho$ determine the decision process. If the subject is in the nonrecognition state, he is more likely to assume that the signal has changed if he was in a recognition state on the foregoing trial. Therefore, $v \geqslant \rho$ is postulated. Whenever $\nu$ and $\rho$ differ from 0.5 , the model predicts sequential dependencies.

\section{SEQUENTIAL PROBABILITIES}

A possible way of discriminating between the two models lies in the comparison of sequential probabilities. In the MT model, the probability of an $A_{1}$ response to an $S_{i}$ presentation following an $S_{k}$ and $A_{j}$ stimulus response pair is given by

$$
p\left(\mathbf{A}_{1} \mid S_{i}, \mathbf{A}_{j}, S_{k}\right)=\Phi\left[\frac{s_{i}-t_{k}-\delta_{j}}{\sigma_{D}}\right],
$$


where $\Phi(x)$ is the cumulative unit normal distribution at argument $x$. Since, $\mathrm{i}, \mathrm{j}$, and $\mathrm{k}$ may be either 1 or 2 , there are eight different sequential probabilities. The sequential probabilities for the MS model may be obtained in a straightforward manner from the transition matrix:

$$
\begin{aligned}
& \mathbf{P}\left(A_{1} \mid S_{1} A_{1} S_{1}\right)=\alpha+(1-\alpha)(\alpha(\rho-\nu)+\nu) \\
& P\left(A_{1} \mid S_{1} A_{1} S_{2}\right)=\alpha+(1-\alpha) \nu \\
& P\left(A_{1} \mid S_{1} A_{2} S_{1}\right)=\alpha+(1-\alpha)(1-\nu) \\
& P\left(A_{1} \mid S_{1} A_{2} S_{2}\right)=\alpha+(1-\alpha)(\alpha(\nu-\rho)-\nu+1) \\
& \mathbf{P}\left(A_{1} \mid S_{2} A_{1} S_{1}\right)=(1-\alpha)(\alpha(\rho-\nu)+\nu) \\
& P\left(A_{1} \mid S_{2} A_{1} S_{2}\right)=(1-\alpha) \nu \\
& P\left(A_{1} \mid S_{2} A_{2} S_{1}\right)=(1-\alpha)(1-\nu) \\
& P\left(A_{1} \mid S_{2} A_{2} S_{2}\right)=(1-\alpha)(\alpha(\nu-\rho)-\nu+1)
\end{aligned}
$$

The first four of the probabilities for each model are hits and the last four are false alarms. Also, due to the stimulus response pair of the preceding trial, each hit rate belongs to one false alarm rate. Therefore, the eight sequential probabilities may be depicted as four points in an ROC plot. By a standard argument, the ROC of the MT model has the familiar bowed shape or, if the probabilities are transformed to unit normal deviates, the ROC is a straight line with unit slope.

From Equations 6, it follows readily that the ROC in hit and false alarm coordinates for the MS model is a straight line with unit slope and intercept equal to $\alpha$.

Since the predicted ROC is different for the two models, this offers one possibility for discriminating between them. Another possibility may be derived from the fact that the relationship between sequential probabilities and response parameters is different for the two models. According to the MT model, each of the sequential probabilities depends on just one of the response parameters, $\delta_{1}$ or $\delta_{2}$. In the MS model, in contrast, four probabilities depend on both $\nu$ and $\rho$. The experiment described below tries to take advantage of this difference.

The idea is to find experimental conditions which influence some of the sequential probabilities and leave others constant. One possibility for achieving this is displayed in Table 1, which shows two payoff conditions used in the experiment.

Under Condition I, each correct answer is rewarded by two points, whereas for each incorrect answer, one point is deducted. Under Condition II, the amount of payoff in addition depends on the last response. This payoff condition should lead to an increase in all sequential probabilities involving $A_{1}$ as preceding response when compared to the corresponding proba-
Table 1

Payoff Conditions I and II

\begin{tabular}{rrrllllll}
\hline \multicolumn{3}{c}{ Condition I } & \multicolumn{5}{c}{ Condition II } \\
& $\mathbf{A}_{1}$ & $\mathbf{A}_{2}$ & & & & $\mathbf{A}_{1}$ & $\mathbf{A}_{2}$ \\
\hline $\mathrm{S}_{1}$ & +2 & -1 & & Last & $\mathbf{A}_{1}$ & +5 & -5 \\
$\mathrm{~S}_{2}$ & -1 & +2 & $\mathrm{~S}_{1}$ & Response & $\mathbf{A}_{2}$ & +2 & -1 \\
& & & $\mathrm{~S}_{2}$ & & & -1 & +2 \\
\hline
\end{tabular}

bilities under Condition I.

The main question is what the two models predict for these two experimental conditions, i.e., which parameters should change and in which direction. The sensory parameters, of course, should remain constant between the two experimental conditions. With respect to the response parameters, on the other hand, the two models make different predictions: In the MT model, an increase in $P\left(A_{1} \mid S_{i} A_{1} S_{k}\right)$ would lead to a decrease in $\delta_{1}$, whereas $\delta_{2}$ should not change. That is, four sequential probabilities should increase from Condition I to Condition II and the remaining four should remain constant. When converted to $\mathrm{z}$ scores, the decrease should be of identical magnitude. In detail, these predictions are:

$z_{I}\left(A_{1} \mid S_{i} A_{1} S_{k}\right)-z_{I I}\left(A_{1} \mid S_{1} A_{1} S_{k}\right)=\delta_{1, I}-\delta_{1, I I}$

$z_{I}\left(A_{1} \mid S_{i} A_{2} S_{k}\right)-z_{I I}\left(A_{1} \mid S_{i} A_{2} S_{k}\right)=0$

where $z_{I}$ and $z_{I I}$ are obtained from the sequential probabilities observed under Conditions $I$ and II after conversion to $\mathrm{z}$ scores and $\delta_{1, \mathrm{I}}$ and $\delta_{1, \mathrm{II}}$ is the response parameter under Conditions I and II, respectively.

The MS model, on the other hand, would account for an increase in $\mathrm{p}\left(\mathbf{A}_{1} \mid \mathrm{S}_{\mathrm{i}} \mathbf{A}_{1} \mathrm{~S}_{\mathrm{k}}\right)$ by an increase in $\nu$ or by an increase in both $\nu$ and $\rho$. Because of Equations 6, the MS model also predicts a rather systematic shift in sequential probabilities between Condition I and Condition II. If the changes in $\nu$ and $\rho$ are denoted by $\mathrm{d}$ and $c$, respectively, then the predictions are:

$$
\begin{aligned}
& P_{I}\left(A_{1} \mid S_{i} A_{j} S_{k}\right)-P_{I I}\left(A_{1} \mid S_{i} A_{j} S_{k}\right) \\
& =\left\{\begin{array}{l}
(1-\alpha)(\alpha(c-d)-c) \\
-(1-\alpha) c \\
(1-\alpha) c \\
(1-\alpha)(\alpha(d-c)+c) \\
(1-\alpha)(\alpha(c-d)-c) \\
-(1-\alpha) c \\
(1-\alpha) c \\
(1-\alpha)(\alpha(d-c)+c)
\end{array}\right.
\end{aligned}
$$


Table 2

Sequential Probabilities: $\mathbf{P}\left(\mathbf{A}_{\mathbf{1}} \mid \mathrm{S}_{\mathbf{i}} \mathbf{A}_{j} \mathbf{S}_{\mathbf{k}}\right)$

\begin{tabular}{lllll}
\hline & & \multicolumn{3}{c}{ Condition } \\
$\mathrm{i}$ & $\mathrm{j}$ & $\mathrm{k}$ & $\mathrm{I}$ & $\mathrm{II}$ \\
\hline 1 & 1 & 1 & .74 & .87 \\
1 & 1 & 2 & .83 & .93 \\
1 & 2 & 1 & .55 & .63 \\
1 & 2 & 2 & .76 & .84 \\
2 & 1 & 1 & .25 & .34 \\
2 & 1 & 2 & .45 & .42 \\
2 & 2 & 1 & .21 & .19 \\
2 & 2 & 2 & .24 & .27 \\
\hline
\end{tabular}

where the subscripts I or II correspond to the payoff conditions. The sequential probabilities appear in the same sequence as in Equations 6 . Note that for every positive difference there is a corresponding decrease of the same magnitude. Furthermore, the absolute differences can take on only one of two possible values.

\section{EXPERIMENT}

\section{Method}

Apparatus. The experimental setup closely followed the Tanner et al. experiment. Signals were two sinusoidal tones of $1,000 \mathrm{~Hz}$. differing in loudness. The louder tone was set to $70 \mathrm{~dB}$ whereas the softer one was individually adjusted for each subject to yitld a probability of correct response of approximately 0.70 . Signals were presented by earphones. The subjects responded by pressing one of two buttons corresponding to the two signals.

Subjects. Ten students served as subjects. They were paid according to their performance as specified by the payoff conditions described in Table 3 . Each point was valued at 1 Pfennig. The subjects were fully informed about the nature of the experimental procedure.

Procedure. Each subject participated in individual sessions under both experinental conditions. There was a total of 10 sessions. 5 under each condition. Because of technical requirements, Condition I in every case preceded Condition II. Since the subjects were highly trained, we assume that no bias was introduced. Each session was divided into 26 series. Each series consisted of 20 trials. Each trial began with a warning light of 1 sec followed by the stimulus presentation of $100 \mathrm{msec}$. Thereatter. the subject had 3 sec time to respond. The a priori probability of $S_{1}$ was 0.5 . During the experiment the subject could look at the payoff matrix drawn on a card in tront of him. The subjects were not given trial-to-trial feedback, but after each series they were told how many points they had earned during the last 20 trials. The different sessions for each subject took place on different days. The whole lirst sessions of each condition as well as the tirst series of all following stessions were viewed as warming-up trials and were excluded from data analysis.

\section{Results and Discussion}

Table 2 contains the empirical sequential probabilities combined for all 10 subjects. Note that the proportion of $A_{1}$ responses has increased from Condition I to Condition II in seven out of eight cases. The overall increase is statistically highly significant, as judged by an $\chi^{2}$ of 304.5 with $1 \mathrm{df}$ obtained from the fourfold table, Type of Response by Condition. Apparently, the subjects reacted to Payoff Condition II by an increased tendency to respond $A_{1}$ regardless of the previous answer. This result was not intended by Condition II, but on the subject's side it appears to be not an unreasonable thing to do. The decisive question now is how the two models can account for this result.

Recall that, in the MT model, an increase in four sequential probabilities would result in a decrease of $\delta_{1}$. The remaining four sequential probabilities determine the value of $\delta_{2}$ independently of $\delta_{1}$. The MS model, on the other hand, predicts that if four sequential probabilities increase, the remaining four should decrease.

To evaluate the two models, parameters were estimated and observed and predicted probabilities were compared. Parameter estimation was done by an iterative routine (Chandler, 1969) which searched for a minimum of the following function

$$
\chi^{2}=\sum_{C=I}^{I I} \sum_{i, j, k, l=1}^{2} \frac{\left[F_{C}\left(A_{i} \mid S_{j} A_{k} S_{l}\right)-\hat{F}_{C}\left(A_{i} \mid S_{j} A_{k} S_{1}\right)\right]^{2}}{\hat{F}_{C}\left(A_{i} \mid S_{j} A_{k} S_{l}\right)},
$$

where $F$ denotes the number of observed and $\hat{F}$ the number of predicted responses under the respective conditions.

Parameters were estimated for each subject individually. The $\chi^{2}$ values are given in Table 3 . The main result is that the MT model agrees much more closely with the data than the MS model does. For 8 out of the 10 subjects, the model does not deviate from the data at the .05 level of significance, whereas the MS model for every subject departs from the data beyond the .01 level.

Figure 1 shows the ROC from four of the subjects. These illustrate that the bow-shaped curve of the MT model accounts better for the data than does a straight line with unit slope as predicted by the MS model.

Figure 1 also shows the result from one of those subjects for which the MT model also did not fit. This failure apparently is caused by the fact that the data points for Condition II moved towards the upper left

Table 3

\begin{tabular}{|c|c|c|}
\hline Subjects & $\begin{array}{l}\text { MT-Mod } \\
\mathrm{df}=26\end{array}$ & $\begin{array}{c}\text { MS-Mod } \\
\mathrm{df}=27\end{array}$ \\
\hline 1 & 19.4 & $273.0^{*}$ \\
\hline 2 & 28.3 & $454.7^{*}$ \\
\hline 3 & 28.5 & $281.5^{*}$ \\
\hline 4 & 14.7 & $63.4^{*}$ \\
\hline 5 & 20.6 & $126.9^{*}$ \\
\hline 6 & 27.7 & $128.0^{*}$ \\
\hline 7 & $101.8 *$ & $138.5^{*}$ \\
\hline 8 & 25.2 & $222.5^{*}$ \\
\hline 9 & 17.6 & $232.2^{*}$ \\
\hline 10 & $93.9 *$ & $113.7^{*}$ \\
\hline
\end{tabular}

Note-Values with $p<.05$ are indicated by an asterisk. 
corner. According to the model, this indicates that this subject responded more sensitively under Condition II. The above parameter estimation, of course, could not account for this change, since the sensitivity parameter was kept constant. Hence, the large $\chi^{2}$ was obtained. The data for Subject 10, which resulted also in a significant $\chi^{2}$, look very similar.

Table 4 gives the parameter estimates for the MT model. The parameter estimates of the MS model are not included because of the bad fit. As can be seen, the MT model accounts for the effect of Condition II by a decrease in both response bias parameters, $\delta_{1}$ and $\delta_{2}$. This is consistent with the interpretation of these parameters. The MS model, on the other hand, could not react in a similar way because of the reciprocal relationship between $\nu$ and $\rho$, as implied by Equations 6 . Thus, the bad fit of the MS model is not caused by the form of the ROC alone, but perhaps to a higher degree by the relationship between the response parameters. One fallacy in Table 4 should be noted, however. For Subject $3, \delta_{1}$ and $\delta_{2}$ appear in reverse order. I have no suggestion on how to explain this except that the search algorithm might have got trapped in a local minimum.

To summarize, in the present study, involving two payoff conditions, the MT model could predict the data much better than the MS model did. This conclusion fits into the results found in signal detection and recognition
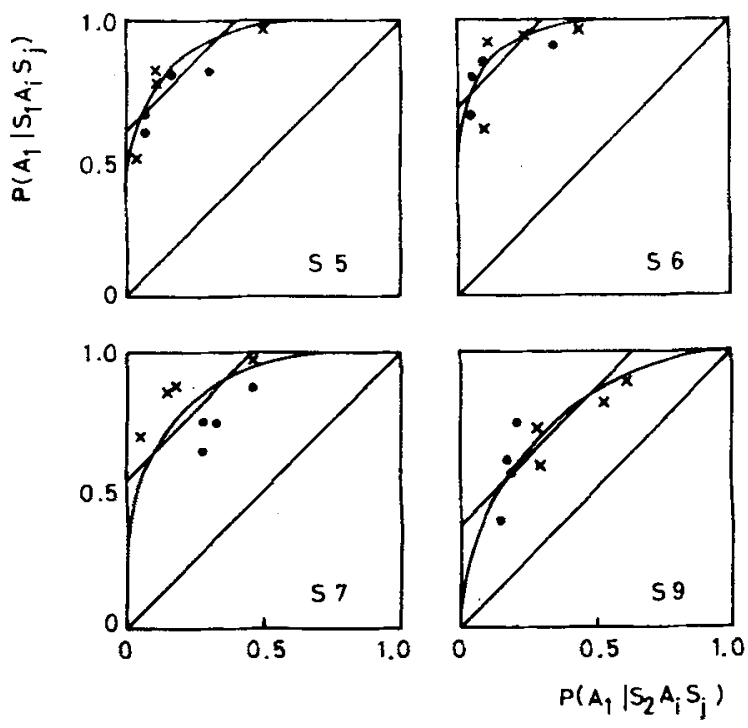

Figure 1. Empirical ROCs from four subjects. Points from Condition I are denoted by - and from Condition II by $\times$. Ako shown are the predicted ROCs [MS model: straight line; MT model: bow-shaped curve].
Table 4

Parameter Estimates

\begin{tabular}{ccrrrrrr}
\hline & & & \multicolumn{2}{c}{ Condition I } & \multicolumn{2}{c}{ Condition II } \\
Subjects & $\alpha$ & $\sigma_{\mathrm{d}}$ & \multicolumn{1}{c}{$\delta_{1}$} & $\delta_{2}$ & $\delta_{1}$ & $\delta_{2}$ \\
\hline 1 & .37 & .75 & -.19 & -.02 & -.54 & -.23 \\
2 & .20 & .66 & -.22 & -.01 & -.69 & -.27 \\
3 & .22 & .81 & -.19 & -.33 & -.34 & -.35 \\
4 & .31 & .97 & -.36 & -.03 & -.37 & +.30 \\
5 & .40 & .54 & +.13 & +.19 & -.19 & +.31 \\
6 & .22 & .44 & -.07 & +.26 & -.30 & +.10 \\
7 & .27 & .67 & -.23 & +.03 & -.24 & +.06 \\
8 & .42 & .64 & -.23 & +.33 & -.56 & -.06 \\
9 & .24 & 1.01 & +.22 & +.49 & -.69 & +.08 \\
10 & .30 & .89 & -.08 & -.03 & -.25 & -.06 \\
\hline
\end{tabular}

memory, where, as mentioned at the outset, continuous models have been found to be superior to discrete threshold models.

\section{REFERENCES}

Blackwell, H. R. Neural theories of simple visual discriminations. Jommal of the Optical Society of America, 1963, 53, 129-160.

Chandler. J. P. STEPIT-Finds local minima of a smouth function of several parameters. Behavioral Science, 1969, 14. $81-82$.

Gorman, A. M. Recognition memory for nouns as a function of abstractness and frequency. Journal of Experimental Psychology, 1961, 61. 23-29.

Krantz. D. H. Threshold theories of signal detection. Psychological Review', 1969, 76, 308-324.

Laming. D. Marhematicul psychology. New York: Academic Press, 1973.

LUCE, R. D. A threshold theory for simple detection experiments. Psychological Review, 1963, 70, 61-79.

Murdock, B. B., Jr. Human memory: Theory and data. Potomac. Md: Erlbaum, 1974.

Parducci, A..\& Sandusky, A. Distribution and sequence effects in judgments. Joumal of Experimental Psychology, 1965, 69. 450-459.

SANDUSKY, A. Signal recognition models compared for random and Markov presentation sequences. Perception \& Psychophysics, 1971, 10, 339-347.

SHEPARD, R. N. Recognition memory for words, sentences, and pictures. Journal of Verbal and Verbal Behavior, 1967, 6. $156-163$.

Tanner. T. A., JR., Haller, R. W., \& Atkinson, R. C. Signal recognition as influenced by presentation schedules. Perception \& Psychophysics, 1967, 2, 349-358.

Tanner, T. A., Jr., Rauk, J. A., \& Atkinson, R. C. Signal recognition as influenced by information feedback. Journal of Mathematical Psychology, 1970, 7, 259-274.

(Received for publication March 1975; accepted July 7,1975 .) 